\title{
AUTOMATION OF GEOMETRY INPUT FOR BUILDING CODE COMPLIANCE CHECK
}

\author{
Ekaterina Petrova ${ }^{1}$, Peter Lind Johansen ${ }^{2}$, Rasmus Lund Jensen ${ }^{3}$, Steffen Maagaard ${ }^{4}$, and \\ Kjeld Svidt ${ }^{5}$
}

\begin{abstract}
Documentation of compliance with the energy performance regulations at the end of the detailed design phase is mandatory for building owners in Denmark. Therefore, besides multidisciplinary input, the building design process requires various iterative analyses, so that the optimal solutions can be identified amongst multiple alternatives.

However, meeting performance criteria is often associated with manual data inputs and retroactive modifications of the design. Due to poor interoperability between the authoring tools and the compliance check program, the processes are redundant and inefficient. That has left the industry in constant pursuit of possibilities for integration of the tool within the Building Information Modelling environment so that the potential provided by the latter can be harvested and the processed can be optimized. This paper presents a solution for automated data extraction from building geometry created in Autodesk Revit and its translation to input for compliance check analysis.
\end{abstract}

Keywords: Building Information Modelling, Building Energy Design, Performance Assessment, Interoperability

\section{INTRODUCTION}

The Energy Performance of Buildings Directive (EPBD) (2002/91/EC and recast 2010/31/ $\mathrm{EU})$ requires all EU members to apply minimum performance requirements for all buildings (The European Parliament and Council 2010). However, besides a framework for the calculation of the overall energy performance, EPBD does not provide a universal application scheme. The strictness of the implementation and all related actions are decided on a national level (The European Parliament and Council 2010).

The Danish Building Regulations (BR15) require calculation of the building's energy demand and documentation of compliance at the end of the detailed design phase. It is performed according to DS418:2011 Calculation of Heat Loss from Buildings, SBi Directive 213 Energy Demand of Buildings, and the adjacent calculation program Be10 based on ISO 13790: 2008 Energy performance of buildings- Calculation of energy use for space heating and cooling (Aggerholm and Grau 2011).

1 PhD Student, Department of Civil Engineering, Aalborg University, Aalborg, Denmark, ep@civil.auu.dk

$2 \quad$ BIM Process Developer, MOE A/S, Aarhus, Denmark, plj@moe.dk

3 Associate Professor, Department of Civil Engineering, Aalborg University, Aalborg, Denmark, rlj@civil.aau.dk

$4 \quad$ Technical Director Energy Design and Indoor Climate, MOE A/S, sem@moe.dk

5 Associate Professor, Department of Civil Engineering, Aalborg University, Aalborg, Denmark, ks@civil.aau.dk 


\section{BACKGROUND}

\subsection{BIM and ISO 13790-based building code compliance checking}

Usually, the early design would not require $100 \%$ accuracy of geometry, and a minimal margin of error is acceptable until later iterations, but for documentation of compliance, a $100 \%$ accuracy is required. Many of the compliance check inputs stem from the building geometry i.e. building location and orientation (including daylight and outdoor climate), building envelope, heating system and hot water supply, heat-accumulating properties, HVAC systems, solar radiation and screening, etc. (Aggerholm and Grau 2011).

However, despite the mandatory use of Be10 for compliance check with both Low Energy Class 2015 (Building Regulations 2015 (BR15)) and Building Class 2020, the geometry input is manual and requires numerous measurements of the building envelope to extract the necessary data. BIM models are a comprehensive source of information, but their effective use is hindered by interoperability issues between authoring tools and Be10. That is valid for both the technical data transfer between the BIM tools and the compliance check program, as well as its translation to analytical input.

\subsection{Research objectives and approach}

The intention of the study is to enable a faster and less resource-intensive compliance check process, and ultimately to support energy performance optimization in the early design. Thus, the primary objective is to foster calculations, where time is not wasted on manual measurements and input in the simulation program. Achieving this would eliminate errors resulting from the manual processes, automate the export of data and make decision support in the early design more efficient. For the professional practice that will result in the actual deployment of the potential of BIM, and will ensure consistency in quantity extraction and applied measurement rules. The study is also a contribution to the interoperability and information management domain and aims to bridge the gap between the industry and academia, concerning building energy design and performance assessment.

The research is conducted under the Design Science Research Methodology (DSRM) (Peffers et al. 2008), which aims for creation and evaluation of an artefact with the purpose of solving particular organizational issues. Despite being conducted in collaboration with one particular organization (MOE A/S design and engineering consultants, the identified issues are characteristic of the industry in general, and a potential solution will have a contribution to all consultancy professionals. A contextual analysis has been performed on the organization's internal energy design workflows by the use of flow models, as defined by Beyer and Holtzblatt (1998). Such an investigation is essential to the research, as it provides an understanding of the processes, actors, information exchanges, and technical systems. Besides using workflow models, interviews, and observations, the authors test three data transfer methods, one of which serves as a basis for the development of the artefact. The latter is tested and evaluated with two cases, which provide the main results of the study. 


\section{ANALYSIS OF THE STANDARD 'AS IS' COLLABORATIVE DESIGN AND COMPLIANCE CHECK WORKFLOW}

The legal norm for use of ICT for project documentation demands the use of a project web in public projects above five million DKK ( $\$ 5$ of ICT order (Klima-, Energi- og Bygningsministeriet 2013)). Each actor uploads a copy of their discipline model to the common platform, which reflects the latest saved version of the model and does not provide an opportunity for tracking of updates. In other words, the information exchange is similar to a document-based one. The process does not prevent the actors from modifying a received model (e.g. adding glass properties to a window object), but the added information is only valid for the particular exchange. As a result, with each model update and new exchange, the same supplementary discipline information has to be added again (Figure 1). That leads to several issues, among which a high level of redundancy.

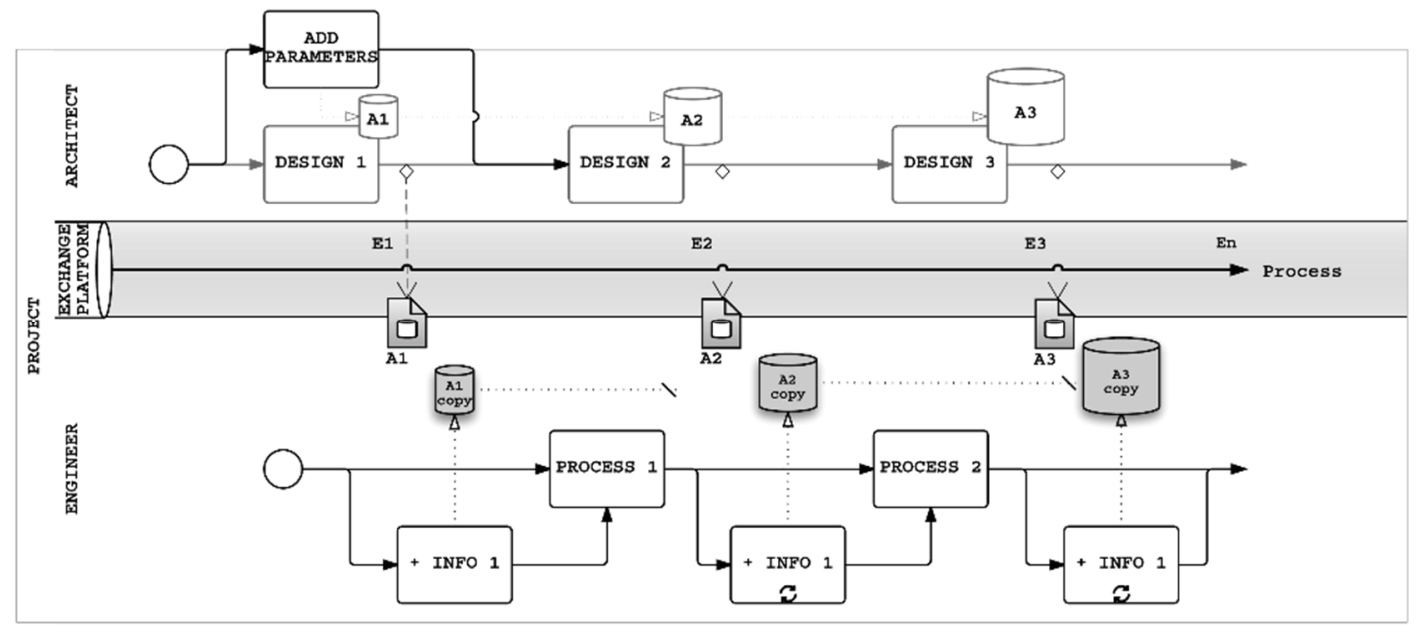

Figure 1: Principle process diagram of discipline model exchange during the design process via the project web, Johansen (2015).

The main reason for the redundancy is the analytical need of object orientation, as the windows' orientation and inclination are particularly important. So, the provision and exchange of that information between the disciplines have been explored in detail (Johansen 2015). The workflow analyses identified a high level of complexity of the 'as is' process and dozens of exchanges associated with the development of the architectural model, adding of inclination and orientation parameters, as well as the performance of the necessary simulations. To solve these issues, it is essential to aim for effective use of the information, which is available in the BIM-model's geometry but is not effectively used in the existing workflow. Therefore, the purpose is to target availability of that information in the sense of automatic export, which can potentially reduce the complexity and eliminate some redundant processes.

\section{INTEROPERABILITY BETWEEN CAD AND BE10}

Being based on the organization's internal workflows, the study requires the use of Autodesk Revit and a solution based on a link between the internal energy analytical template and the architectural model (i.e. by the use of Revit's link function). Three individual tests were completed with (1) IFC (an international standard for data exchange) (buildingSMART, 2015), (2) gbXML (a preferred exchange standard for energy analysis) 
(Stumpf et al. 2011), and (3) Revit Application Programming Interface (API). The evaluation is based on a set of criteria, listed according to priority.

\subsection{Mapping of criteria}

The evaluation criteria are determined in collaboration with the professionals and are based on their knowledge, experience, and assessment of potential value. The prioritization, where 1 is Highest and 7 is Lowest priority, is as follows:

1. Reliability: Provides valid data/results- $100 \%$ correct geometry

2. Value: Process optimization rate

3. Availability: Data processing in Excel

4. Process: Compatibility with BIM-data relevant for Be10

5. Future proofing: Forward-looking development

6. Implementation: Automate activities with the least amount of steps

7. Bi-directional exchange: Potential for import of Excel/Be10-data

Prioritized are also Input (I) and Output data (O), and their association with BIMGeometry $(\mathrm{G})$ or Property (P), where (1) is High and (2) Medium Priority (Table 1).

Table 1: Be10 parameters according to priority, Johansen (2015)

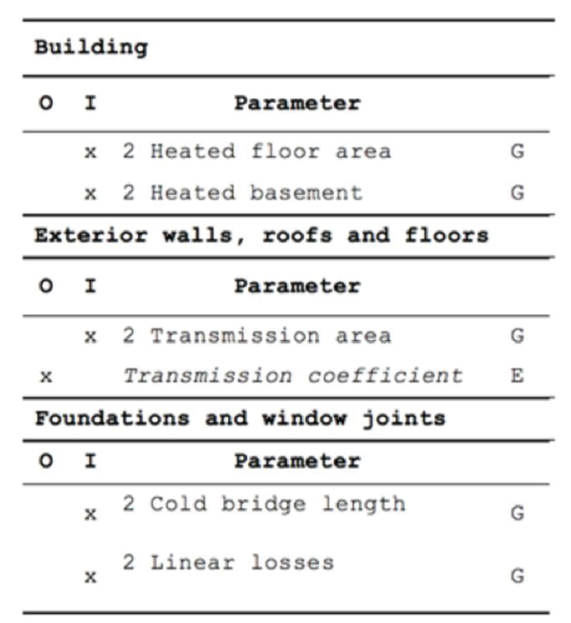

\begin{tabular}{|c|c|c|c|}
\hline \multicolumn{4}{|c|}{ Windows and exterior doors } \\
\hline o & I & Parameter & \\
\hline & $\mathbf{x}$ & 1 Amount & G \\
\hline & $\mathbf{x}$ & 1 Orientation & G \\
\hline & $\mathbf{x}$ & 1 Inclination & G \\
\hline & $\mathbf{x}$ & 1 Transmission area & G \\
\hline $\mathrm{x}$ & & Transmission coefficient & $\mathrm{E}$ \\
\hline \multicolumn{4}{|c|}{ Internal heat gain } \\
\hline \multirow[t]{5}{*}{0} & I & Parameter & \\
\hline & $\mathrm{x}$ & 2 Area & G \\
\hline & & 2 People $\left[\mathrm{W} / \mathrm{m}^{2}\right]$ & E \\
\hline & $\mathrm{x}$ & 2 Equipment OPHR $\left[\mathrm{W} / \mathrm{m}^{2}\right]$ & $\mathrm{E}$ \\
\hline & $x$ & $\begin{array}{l}2 \text { Equipment outside } \\
\text { OPHR }\left[\mathrm{W} / \mathrm{m}^{2}\right]\end{array}$ & $\mathrm{E}$ \\
\hline
\end{tabular}

\subsection{Test case}

The exchange methods are tested individually by the use of a model specifically developed for the test (Figure 2). Be10 determines orientation according to North corresponding to $0^{\circ}$ and increasing clockwise (Aggerholm and Grau 2011). The orientations in the study are calculated according to the Cartesian system (i.e. degrees increasing counter-clockwise). Thus, each mathematically determined window orientation functions as a reference value and not as an actual input value in Be10. 

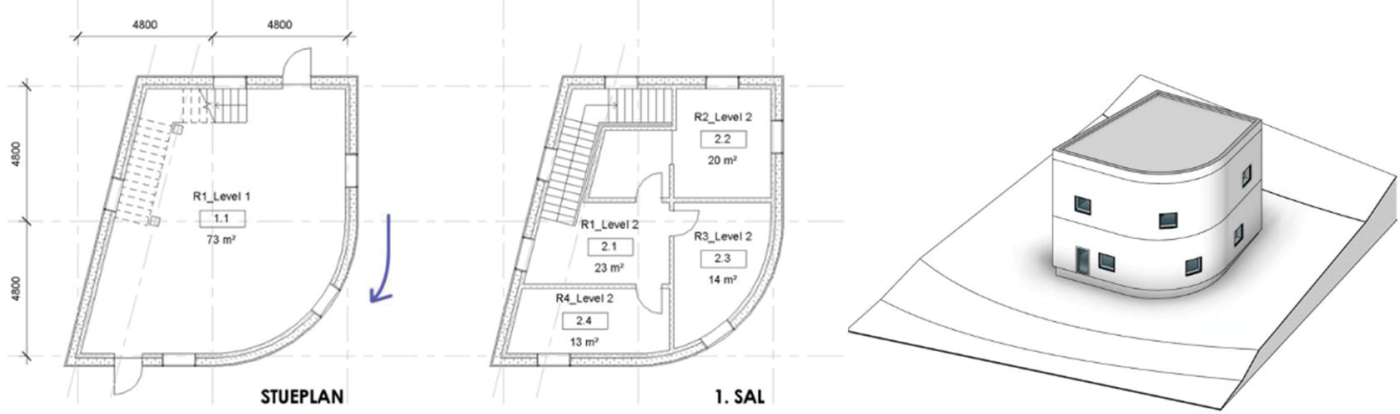

Figure 2: Plan views and isometric view of the test model, Johansen (2015).

\subsection{Test of methods for geometry export}

\subsection{1 gbXML}

The export to gbXML is based on a generated Revit Energy Analytical Model (EAM). According to Autodesk Inc. (2015), at the time of the study openings in curved walls are not supported, which, considering the model's geometry may be a determining factor, even though the preview does not indicate any immediate problems. That is valid for setup with parameters Building Type: Office, Include Thermal Properties, Building Envelope: Use Function Parameter, Export Category: Rooms. However, changing the export category to Analytical Surfaces confirms the issue, as the placement of the windows, which is relative to the export category does not change.

BIM-objects are given a function code, with each room as a supertype of the bounding elements, which by themselves function as supertypes for openings in walls. The codes are given a function, type (e.g. $\mathrm{E}$ and $\mathrm{W}$ for direction) and a running number for space boundary, and are inherited from supertype to subtype. Walls bounding more than one rooms contain the code of each of them, e.g. N-21-22-E-W-15. All building elements are thus given orientation. This also applies to the curved walls, at which point the consequence of the lack of support becomes more prominent. Revit divides the curved walls are into several small ones, and that division is also inherited by the window, which is thus exported as four separate windows.

\subsubsection{IFC}

IFC Exporter for Revit 2015 (version 15.2.0) has been used for the export, with a custom export for Concept Design BIM 2010 (CDB-2010) - the superior Model View Definition for Nordic Energy Analysis (NOW-001) (buildingSMART 2013). "Store the IFC GUID as an element parameter after export" has been selected to ensure that the objects' GUID (Globally Unique Identifier) is: (1) visible in the property data in Revit and (2) will remain unchanged even after several exports.

DDS-Cad Viewer confirms that the IFC-model is able to handle the geometry. IFC File Analyzer (Lipman 2014) is further used for generation of an Excel spreadsheet with sorted data according to the entities. Including information on placement and orientation is done with ifcLocalPlacement and ifcAxistoPlacement selected. The generated spreadsheet shows that the IFC model handles windows in the curved objects as intended, as the extracted windows (12) equal the amount modelled. A comparison of the generated spreadsheet data with the test model geometry in Revit is presented in Johansen (2015).

ifcWallStandardCase, ifcOpeningElement and ifcWindow have been further examined in the curved and straight walls. In the case of the straight wall, Location for all three defines the relative placement of the entity's origin in relation to the hosting entity's origin. 
Direction is defined by $\mathrm{x}$ and $\mathrm{y}$-coordinates (RefDirection) and vertical inclination- by $\mathrm{z}$. The direction is determined according to the axes of a two-dimensional Cartesian system by identifying the quadrant $\mathrm{x}$ and $\mathrm{y}$ are located in, and calculation of $\tan ^{-1}$ in order to convert Cartesian to Polar coordinates. A detailed conversion for all objects is presented in Johansen (2015). With regards to the curved wall, Location appears in the data for all three (ifcWallStandardCase, ifcOpeningElement and ifcWindow), but RefDirection is only defined for the window. Therefore, its direction is determined the same way as previously described. Even though RefDirection values vary, they are relatively uniform, which may be due to the test model being relatively simple and the facades being modelled in a clockwise direction. The modelling direction, however, does not have an effect on the orientation of the windows (Johansen 2015).

\subsubsection{Revit Application Programming Interface}

To perform this test, the authors used Revit 2015, Update Release 4. Revit Lookup is used for viewing and navigating element properties and relationships in the project database. The SnoopDB function lists the classes included in the Revit project. Windows and doors are listed under FamilyInstance and marking each FamilyInstance or occurrence makes it possible to view supplementary information. Information related to the windows is divided into three categories, i.e. Element, Instance and FamilyInstance. Data from a geometric Revit object is extracted with the help of Methods/Properties of the Classes. Limited information about the object(s), which have been selected in the project may be accessed with 'Snoop Current Selection'. With this feature, it was possible to review the data related to the same windows, which have been on focus until now.

FamilyInstance contains information about the west façade window's orientation and spatial relations, which are highly relevant to the energy performance analyses, namely a vector and an opening direction with $\mathrm{x}, \mathrm{y}$, and $\mathrm{z}$ coordinates in respectively 'Facing orientation' and 'Hand orientation'. The point of intersection for the Location coordinates, in this case, is placed in the middle of the window and indicates absolute placement, which is contrary to the relative one in the IFC model. The calculations for verification of the direction show a result consistent with the one from the IFC test (Johansen, 2015).

With regards to the window in the curved façade wall, information about Location, Rotation, and Orientation appears to be similar. Information about related objects (hosts, etc.) is accessible, but the location and orientation are specified in a unique way.

\section{RESULTS}

\subsection{Observations}

The decisive factor in the assessment of gbXML as a data exchange method is the Revit EAM, which generates the model for export. The fragmentation of the window is a problem that stands out and creates false results. With regards to orientation, results show that every object is enriched with the relevant information, but as a result of the problem with EAM, it is impossible to conclude whether gbXML is suitable for information transfer to Be10 when a point of departure is Revit. Therefore, based on all observations, and considering that compliance requires $100 \%$ precision of exported geometry, this method is deemed inapplicable and will not be further considered.

The information contained in the IFC model is available and to a great extent readable by following the objects' references to each other. That also applies to the defined geometry, including windows and orientations. The direction of the object types is stored, 
and the information is associated with a logical object, consistent with the hierarchy in Spatial Structure and relative placement. XYZ values are defined only where necessary. In the analysis of IFC, the focus has been put on Location and ObjectPlacement, which in this case is the wall's centreline. With regards to Be10, this would be insufficient, as it requires gross areas (Aggerholm and Grau 2011). As a result, defined space boundaries can be related to extraction from other parts of the IFC model.

FacingOrientation in the API is specifically intended to make the orientation of FamilyInstances available. The direct access to the XYZ values means that the numbers are generated by the system itself and reflect the information in the geometry. An output based on these values depends, therefore, on less complex calculations with other available geometric data. However, the basis for area calculation of the windows was difficult to identify.

\subsection{Evaluation}

Based on the observations, it can immediately be concluded that the export to gbXML provides misleading output. The encountered geometry fragmentation of the window in the curved wall is most likely caused by Revit EAM.

When it comes to extraction of information related to orientation and inclination, both IFC and Revit API are deemed suitable, but at the same time, both require further analyses in relation of extraction of reliable areas. Considering that Be10 requires gross areas for the calculations, IFC's specifics in relation to space boundaries may present issues. Valuable advantages of Revit API are the bi-directional communication and unambiguous information about orientation.

In consideration have been taken the holistic potential for process optimization, specifics of the collaborative processes and potential for implementation in relation to the processes, of which Revit is an important element. The samples in Revit SDK show versatility in functionality related to development in Revit API. Conover (2011) also describes further opportunities in relation to definition of space boundaries.

The latter, combined with the possibility for implementation directly in Revit's interface create a potential for the API, which is outside of IFC's functional area. Based on all considerations, the presented solution is in the form of an Add-in for Revit 2015.

\section{ARTEFACT DEVELOPMENT AND DEMONSTRATION}

To be able to validate the suitability of the chosen method, a number of goals for the abilities of the solution were stated. These include (1) Extraction of relevant information on orientation and inclination, (2) Data extraction from the linked Revit model, (3) Export to Excel, and (4) Usable output without a need for manual adjustment.

The add-in has been developed in steps by gradually expanding functionally, to finally implement the function for export to Excel. Along the way, data was being shown in Revit's UI in order to verify the information that has been accessed through the code. Table 2 presents the general development stages. Each step is associated with numerous processes, conversions, and reviews to ensure usability and readability of the data in relation to the stated goals. Implementation in full detail is described in Johansen (2015).

\subsection{Testing}

The functionality and output have been tested with two cases- an adjusted version of the model previously used, as well as a new, more complex model. Figure 3.1 shows the results from the test with the previously used and adjusted model. Adjustment includes 
modification of the roof from flat with $3^{\circ}$ slope to a hipped one with slopes of 30, 50 and $65^{\circ}$ respectively. Five skylights have been added to the four roof hips. The purpose is to allow checking of the validity of the calculations related to the inclination of the windows.

Table 2: Programming steps of the Revit add-in development, Johansen (2015).

\begin{tabular}{cc}
\hline Accessed information & Data representation in the UI \\
\hline FamilyInstances & Name and ID \\
'Mark'-parameter & Parameter value \\
FacingOrientation & XYZ \\
Area & Area in $\mathrm{ft}^{2}$ \\
Linked Revit model & File name \\
FamilyInstances in model-link & Name, ID, 'Mark', XYZ, Area in $\mathrm{ft}^{2}$ \\
MS Excel export & Confirmation for completion, sum of the \\
processed window objects, duration
\end{tabular}

The results show that the Excel input is formatted according to the data type for every parameter. This makes it possible to use the orientation and inclination values without manual adjustments. To check validity, all values relevant to the Be10 calculations were checked by obtaining the same results manually (Johansen, 2015).

The used test model, however, was built with the specific purpose to undergo the test. It is thus not representative of cases affected by unforeseen circumstances resulting from project scale or modelling issues. Therefore, a test with an architectural model from an actual project was also conducted. The building has 107 windows in total, distributed over facades with diverse angles. The new model is linked to the same project as the previous test model, so in this case, windows from both models are exported (Figure 3.2).

The grey cells in Figure 3.2 have been validated by manual measurements in the architectural model. The skylights in the new model do not have a slope and inclination for them appears as $0^{\circ}$ in Excel. That implies that the program delivers output as intended.

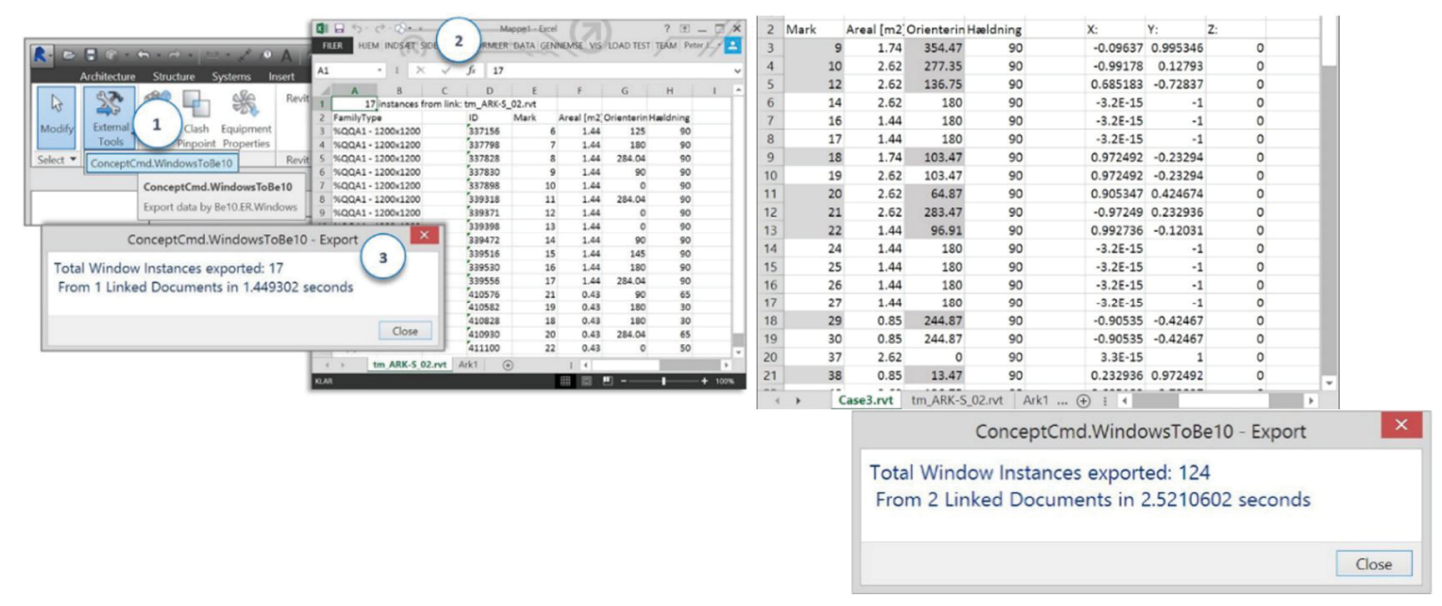

Figure 3.1(left) and 3.2 (right): Activation, output to Excel and final dialog boxes for the two exports, Johansen (2015). 


\section{DISCUSSION}

The add-in provided correct results for orientation and inclination. However, it is a solution, which requires further development. For instance, it will generate empty spreadsheets if a project does not contain a linked Revit model and an error will appear if a model is not loaded. Another issue is the calculation of window areas based on the API, which at the time being is unexplained. The output is correct, but for simple rectangular windows. The code will run for windows with alternative shapes, but the output may be misleading. An error message could potentially solve that issue. Such considerations are an integrated part of automation processes and are a good basis for further development. The program still needs to be able to adjust for rotated true North but has the potential to minimize redundancy by eliminating the need for local copies. An export of 124 windows in 21.2 seconds is a result, which manual measurement cannot conquer, regardless of experience and insight.

\section{CONCLUSION}

While trying to make use of the available technology, the energy frame compliance check required by the Danish Building Regulations is hindered by restrictions in it. These restrictions create a mismatch between the object-oriented BIM principles and the actual work processes. The workflow analysis identified that window orientation is of fundamental importance to the energy performance calculations and is the reason for complex procedures, resulting from the missing direct link between authoring tools and the compliance check program.

Therefore, the study targets the automation of data extraction from building geometry and its translation to input for the building performance assessment. Conducted under the Design Science Research Methodology, the study's purpose is to develop an artefact, which will facilitate solving of the identified issues. The process analyses have argued strongly for focusing on window parameters, and therefore these have been of highest priority in the various information exchange tests. The latter, together with the related analyses show that the sources of the issues extend beyond the single data exchange method. The problem is particularly related to the export with gbXML but is grounded in Autodesk Revit, the use of which is a prerequisite. That by itself underlines the importance of the authoring tools and the effect they may have on the entire process. The test concludes with deeming both IFC and Revit's API being able to provide the relevant information. Based on the compliance check program's analytical needs and Revit being an important element, an API- development was found to have the biggest potential.

The solution that this paper presents is a Revit add-in for geometry export to Excel, which has been able to deliver valid results. Despite the need of further development, its ability to transfer the essential information about object orientation can have a positive impact on the project organisation and thereby eliminate many of the associated manual processes.

\section{REFERENCES}

Aggerholm, S. and Grau, K. (2011). Bygningers Energibehov, Hørsholm: Statens Byggeforskningsinstitut, Aalborg Universitet. Autodesk Inc. (2015). Energy Analysis Using Building Models-Model Validation. 
[https://knowledge.autodesk.com/support/revit-products/learnexplore/caas/CloudHelp/cloudhelp/2015/ENU/Revit-Analyze/files/GUID-9DB01490A0A1-4DA2-A9A9-4F766958017A-htm.html [Senest hentet eller vist den 15 januar 2017].

Beyer, H. and Holtzblatt, K. (1998). Contextual Design: Defining Customer-Centered Systems. San Francisco, CA: Morgan Kaufmann Publishers.

buildingSMART. (2015). IFC Overview Summary. [Online] Available at: http://www.buildingsmart-tech.org/epecifications/ifc-overview

buildingSMART. (2013). Overview of Information Delivery Manuals independent of their status. [Online] Available at: http://iug.buildingsmart.org/idms/overview

Conover, S. (2011). Geometric Progression: Further Analysis of Geometry using the Autodesk Revit 2012 API. s.l.:Autodesk University.

Johansen, P.L. (2015). BIM og Energianalyse - Optimering af Ingeniørens Modelleringsprocesser. Aalborg University.

Klima-, Energi- og Bygningsministeriet. (2013). Bekendtgørelse om anvendelse af informations- og kommunikationsteknologi i offentligt byggeri - retsinformation.dk. https://www.retsinformation.dk/Forms/R0710.aspx?id=145421\&exp=1

Leavitt, H. J., and Bahrami, H. (1988). Managerial psychology: managing behavior in organizations. Chicago, University of Chicago Press.

Peffers, K., Tuunanen, T., Rothenberger, M. and Chatterjee, S. (2008). A Design Science Research Methodology for Information Systems Research. Journal of Management Information Systems. 24(3), pp.45-77, DOI 10.2753MIS0742-1222240302.

Stumpf, A. L., Kim, H. and Jenicek, E. M. (2011). Early Design Energy Analysis Using Building Information Modeling Technology, Champaign, IL: US Army Engineer Research and Development Center.

The European Parliament and Council. (2010). Directive 2010/31/EU of the European Parliament and of the Council of 19 May 2010 on the Energy Performance of Buildings (recast). Official Journal of the European Union 18.6.2010. 